Camila Carvalho de Sousa ${ }^{a}$

(iD) https://orcid.org/0000-0002-6139-0984

Tânia Maria de Araújo

(iD) https://orcid.org/0000-0003-2766-7799

Paloma de Sousa Pinho ${ }^{b}$

(iD) https://orcid.org/0000-0001-6402-0869

Aline Macedo Carvalho Freitas ${ }^{\mathrm{a}}$

(iD) https://orcid.org/0000-0002-5696-9625

a Universidade Estadual de Feira de Santana (UEFS), Núcleo de Epidemiologia, Programa de Pós-Graduação em Saúde Coletiva (DSAU/ UEFS). Feira de Santana, BA, Brasil.

b Universidade Federal do Recôncavo da Bahia (UFRB), Centro de Ciências da Saúde (CCS). Santo Antônio de Jesus, BA, Brasil.

Contato:

Camila Carvalho de Sousa

E-mail:

sousa.camila@ebserh.gov.br

Este estudo foi financiado por: Fundação de Amparo à Pesquisa do Estado da Bahia (Fapesb) (Processos $n^{\circ}$ SUS0024/2009 e no SUS0022/2014) e Coordenação de Aperfeiçoamento de Pessoal de Nível Superior (Capes) com a concessão de bolsa de mestrado (Número do Pedido: 11596/2015).

Os autores declaram que não há conflitos de interesses.

Os autores informam que o trabalho não foi apresentado em evento científico.

Baseado na dissertação de mestrado Insatisfação com o trabalho entre trabalhadores de saúde e ocorrência de transtornos mentais comuns, de Camila Carvalho de Sousa, defendida em 2017. na Universidade Estadual de Feira de Santana, BA, Brasil.

Recebido: 05/06/2018

Revisado: 12/02/2019

Aprovado: 15/04/2019

\section{Insatisfação com o trabalho em saúde: fatores associados e diferenciais de gênero}

\author{
Dissatisfaction with health work: \\ associated factors and gender differentials
}

\section{Resumo}

Objetivo: avaliar fatores associados à insatisfação com o trabalho em Saúde, segundo gênero. Métodos: estudo transversal, exploratório, conduzido entre 2011-2012, na Bahia, com 3.084 trabalhadores/as da saúde. A insatisfação com o trabalho foi avaliada segundo características sociodemográficas e laborais por meio de análises bivariadas e multivariadas, estratificadas por sexo. Resultados: a insatisfação com o trabalho entre homens foi de 27,4\%, entre mulheres, 25,6\%. Nas mulheres, mostraram-se associadas à insatisfação: idade até 40 anos (razão de prevalência - RP: 1,36; intervalo de confiança de 95\% - IC 95\%: 1,17-1,58), nível de escolaridade técnico (RP: 0,70; IC 95\%: 0,58-0,86), e médio/fundamental (RP: 0,66; IC 95\%: 0,57-0,77), vínculo de trabalho temporário (RP: 0,66; IC 95\%: 0,56-0,77) e exercer atividades não compatíveis com o cargo (RP: 2,17; IC 95\%: 1,81-2,60). Para os homens: idade até 40 anos (RP: 2,09; IC 95\%: 1,56-2,81), exercer atividades não compatíveis com o cargo (RP:1,74; IC 95\%: 1,30-2,34) e vínculo de trabalho temporário (RP:0,57; IC 95\%: 0,44 - 0,74). Discussão: homens e mulheres vivenciam elevados níveis de insatisfação com o trabalho, com características sociodemográficas e laborais associadas. A escolaridade se mostrou inversa entre os estratos, revelando desigualdades de gênero. Conclusão: diferenças de gênero impactam os níveis de insatisfação com o trabalho e é relevante considerá-las para elevar os níveis de satisfação no trabalho.

Palavras-chave: condições de trabalho; gênero; satisfação no emprego; saúde do trabalhador.

\begin{abstract}
Objective: to evaluate factors associated with job dissatisfaction among health workers, according to gender. Methods: we conducted a cross-sectional, exploratory study, from 2011 to 2012, in Bahia State, Brazil, including 3,084 health workers. We evaluated job dissatisfaction according to sociodemographic and labor characteristics through bivariate and multivariate analyses, stratified by gender. Results: the dissatisfaction rate was $27.4 \%$ among men, and $25.6 \%$ among women. The following were shown as being associated with dissatisfaction among women: age up to 40 years (prevalence ratio - PR: 1.36; confidence interval 95\% CI: 1.17-1.58), technical (PR: 0.70; 95\%CI: 0.58-0.86) and elementary/high school educational level (PR: 0.66; 95\%CI: 0.57-0.77), temporary employment contracts (PR: 0.66; 95\%CI: 0.56-0.77) and being engaged in activities not compatible with their position (PR: 2.17; 95\%CI: 1.81-2.60). For men: age up to 40 years (PR: 2.09; 95\%CI: 1.56-2.81), being engaged in activities not compatible with their position (PR: 1.74; 95\%CI: 1.30-2.34) and temporary employment contract (PR: 0.57; 95\%CI: 0.44-0.74). Discussion: men and women experience high levels of job dissatisfaction, which were associated with sociodemographic and work characteristics. Educational level was the opposite between gender strata, revealing the persistence of gender inequalities. Conclusion: gender differences affect job dissatisfaction levels, and it becomes relevant to take them into consideration to raise the levels of job satisfaction.
\end{abstract}

Keywords: working conditions; gender; job satisfaction; occupational health. 


\section{Introdução}

A crescente inserção da mulher no mercado de trabalho, observada nos últimos anos, não foi suficiente para minimizar as desigualdades relativas ao gênero no ambiente laboral. Assimetrias relacionadas às oportunidades de emprego e às diferenças salariais, bem como a possibilidade de ascender na profissão e a desvalorização das funções majoritariamente exercidas por mulheres ainda persistem, com comprovada influência nos níveis de satisfação com o trabalho entre homens e mulheres ${ }^{1}$.

Considerada uma emoção positiva de bem-estar, a satisfação com o trabalho resulta da avaliação que o/a trabalhador/a faz sobre o seu trabalho e da realização de seus valores por meio da atividade que executa. A insatisfação com o trabalho, por sua vez, corresponde a um estado emocional desagradável resultante da avaliação que o indivíduo faz sobre o seu trabalho ou da impossibilidade de realização de seus valores, desejos e motivações pessoais ${ }^{2}$.

No contexto ocupacional, os/as trabalhadores/ as comparam suas necessidades e desejos aos incentivos, vantagens ou direitos adquiridos por meio das atividades (expectativa versus realidade), tais como segurança, estabilidade no emprego, ambiente saudável, possibilidade de crescimento na carreira e apoio emocional. Quando se aproximam dos desejos dos/as trabalhadores/as, os incentivos organizacionais tornam real o aumento da satisfação no trabalho. Isso resulta em menor carga psíquica demandada na realização das atividades e, consequentemente, menor evidência de estresse ocupacional e melhor desempenho e eficiência das funções proporcionadas pela relação de prazer e bem-estar vivenciada no trabalho ${ }^{3}$.

Estudos apontam que as mulheres são mais satisfeitas com o trabalho do que os homens ${ }^{4,5}$. Como explicação para isto, tem sido defendida a hipótese de que a satisfação com o trabalho é um fator de construção social do gênero feminino ${ }^{6}$, que reproduz a concepção de docilidade das mulheres como algo da natureza e a ideia de que elas nutrem menores expectativas em relação ao trabalho do que os homens, sentindo-se por isso satisfeitas mais facilmente ${ }^{7}$.

Em oposição, tem-se encontrado evidências de que não há diferenças significativas nos níveis de satisfação entre homens e mulheres ${ }^{8-10}$. Mas os dados empíricos são ainda controversos. Estudo realizado por Bacha e colaboradores, por exemplo, revelou que os homens eram mais satisfeitos com o trabalho do que as mulheres ${ }^{1}$.

Como características do trabalho de homens e mulheres diferem, os aspectos relativos à satisfação têm apresentado disparidades de gênero. Por exemplo, os homens estão mais satisfeitos com a carga horária de trabalho e com a oportunidade de ascensão na profissão; em contrapartida, as mulheres encontram-se mais satisfeitas com a relação interpessoal da equipe e com a contribuição social do trabalho. Assim, percebe-se que os aspectos mais objetivos do trabalho (como horas e carga de trabalho) satisfazem mais aos homens, enquanto as mulheres estão mais satisfeitas com os fatores mais relacionais (como relação interpessoal e contribuição social) ${ }^{11}$. Salienta-se, contudo, que as diferenças relativas à satisfação com o trabalho entre gêneros desaparecem entre os/as trabalhadores/as jovens e com maior nível de escolaridade ${ }^{7}$.

Percebe-se que não há consenso sobre níveis de satisfação com trabalho quando se avaliam aspectos relativos ao gênero. As características do tipo de trabalho desempenhado e as condições nas quais ele é executado podem explicar esses diferenciais observados, inicialmente contraditórios. De qualquer modo, dada a heterogeneidade de resultados obtidos, a maior exploração desses dados continua sendo um campo aberto para investigação.

Considerando a divisão sexual do trabalho como algo que estrutura as relações laborais, as quais, por sua vez, estruturam experiências distintas nos níveis de satisfação com trabalho, torna-se pertinente a análise da insatisfação à luz das relações de gênero, de modo a avaliar melhor os aspectos que contribuem para a insatisfação com o trabalho. Este estudo objetivou avaliar os fatores associados à insatisfação com o trabalho entre trabalhadores/as de saúde, segundo gênero.

Investigar a satisfação com o trabalho em saúde, considerando as particularidades existentes entre homens e mulheres, pode contribuir para a identificação de problemas nos serviços e para o planejamento de ações que podem elevar os níveis de satisfação, visando a saúde e o bem-estar desses/as trabalhadores/ as e melhorias no ambiente laboral e na qualidade dos serviços prestados. Isto pode contribuir para o fortalecimento do Sistema Único de Saúde (SUS), pois acredita-se que, à medida que se sentirem mais satisfeitos/as, os/os trabalhadores/as poderão, por sua vez, valorizar mais as ações que executam, elevando o seu nível de comprometimento com a qualidade da assistência.

\section{Métodos}

Trata-se de estudo epidemiológico de corte transversal que integra um projeto multicêntrico de investigação das condições de trabalho, condições de emprego e saúde dos trabalhadores da saúde na Bahia, conduzido em cinco municípios baianos (Ilhéus, Feira de Santana, Jequié, Salvador e Santo Antônio de Jesus), com trabalhadores/as de saúde dos serviços de média complexidade e da atenção básica, realizado entre os anos de 2011 e 2012.

A definição da população de estudo foi realizada por meio de levantamento prévio dos/as trabalhadores/as junto às secretarias municipais de saúde. Delimitou-se o número e o tipo de serviços de saúde disponíveis, o número de trabalhadores/as, o sexo, suas respectivas ocupações, bem como a área geográfica em que cada serviço se localizava. A amostra foi constituída por amostragem aleatória, representativa da população, estratificada por: área geográfica (regional), nível de complexidade existente na 
rede (atenção básica e média complexidade), grupo ocupacional e sexo. Para cálculo do tamanho amostral do estudo multicêntrico, assumiu-se prevalência do evento de interesse de $42,6 \%$, erro absoluto de 4,2 e nível de confiança de 95\%. A partir desses parâmetros chegou-se a uma amostra inicial de 515 trabalhadores. Admitindo-se perdas e recusas em torno de $20 \%$, a amostra necessária foi de 618 trabalhadores/as. O estudo alcançou 3.084 indivíduos.

Para verificar se a amostra alcançada pelo estudo multicêntrico, do qual o presente estudo faz parte, tinha poder para avaliar prevalência e os fatores associados à insatisfação com o trabalho em saúde, uma vez que a amostra não havia sido calculada com essa finalidade, o tamanho amostral foi recalculado, com a utilização do software OpenEpi, versão 3.03a. Utilizou-se como parâmetro estudo realizado com trabalhadores/as da atenção básica ${ }^{12}$, devido às similaridades desse grupo de trabalhadores/as, apesar das diferenças regionais. Considerando a prevalência do evento de interesse de $44,9 \%$, nível de confiança de $95 \%$, erro absoluto de $4 \%$ e acrescentando-se a estimativa de $20 \%$ para perdas/recusas, obteve-se o número estimado para compor a amostra de 627 trabalhadores/as. Como foram investigados vários fatores, tomou-se como parâmetro a variável "vínculo empregatício"13, que definiu o maior número de trabalhadores/as a serem estudados. Adotaram-se como parâmetros: prevalência entre expostos $(25,3 \%)$; prevalência em não expostos $(38,2 \%)$; razão não expostos/ expostos (2); nível de significância (95\%); e poder (90\%), o que totalizou 701 trabalhadores/as, já adicionando $20 \%$ a mais a fim de compensar não respostas e perdas. Portanto, o número de trabalhadores/as investigados/as pelo estudo multicêntrico (3.084) foi superior ao necessário à análise desejada.

A coleta de dados foi realizada por meio de questionário estruturado. Para padronizar os procedimentos metodológicos adotados em cada local, foi elaborado um manual de procedimentos e condutas para pesquisa de campo e realizadas oficinas para treinamento dos/as entrevistadores/as.

Realizou-se estudo piloto em uma cidade baiana, com 30 trabalhadores da atenção básica, com o objetivo de treinar os/as pesquisadores/as para o trabalho de campo, padronizar a abordagem dos indivíduos na coleta dos dados, garantir o preenchimento adequado do questionário e avaliar a viabilidade do instrumento de pesquisa.

A variável desfecho foi a insatisfação com o trabalho, avaliada por meio do seguinte questionamento: "Você está satisfeito/a com o seu trabalho?". As opções de resposta incluíram uma escala tipo Likert, que variou de 1 a 4 , sendo possíveis as seguintes respostas: "1. Não estou satisfeito/a de forma nenhuma"; "2. Não estou satisfeito/a"; "3. Estou satisfeito/a"; e "4. Estou muito satisfeito/a". Optou-se por dicotomizar as respostas em satisfeitos/as e insatisfeitos/as, visto que as frequências obtidas nas quatro opções da escala variaram substancialmente, havendo opção com frequência muito baixa. Assim, foram considerados insatisfeitos aqueles/as que responderem 1 ou 2 e satisfeitos/as aqueles/as que responderam 3 ou 4.

A utilização desse tipo de escala para avaliar a insatisfação é possível pelo fato de satisfação e insatisfação representarem sentidos opostos de um mesmo fenômeno ${ }^{9}$. Sabe-se que a satisfação com o trabalho é influenciada por múltiplos fatores, devendo ser considerada a multicausalidade dos eventos que a determinam. Contudo, estudos têm demonstrado a viabilidade de medir a satisfação com o trabalho com uma única questão. Abordagens desse tipo apresentaram correlação positiva com as medidas realizadas a partir de questionários multidimensionais - mais de uma questão ${ }^{14}$.

A utilização de questão única para estimar a satisfação com o trabalho apresenta vantagens, tais como: tem melhor custo-benefício, pode conter maior validade, é melhor em capturar mudanças na satisfação com o trabalho ${ }^{15}$.

A insatisfação com o trabalho foi analisada segundo características sociodemográficas (sexo, idade, cor da pele, situação conjugal, filhos, renda) e informações gerais sobre o trabalho (função, carga horária semanal, turno de trabalho, vínculo de trabalho, tempo de trabalho na profissão, tempo de trabalho na unidade, realização de atividades compatíveis com o cargo, direitos trabalhistas).

Realizou-se análise estratificada por sexo, na tentativa de descrever diferenças ou similaridades no padrão de distribuição das variáveis que influenciam a insatisfação com o trabalho entre homens e mulheres. Foram realizadas análises bivariada e multivariada. Na bivariada foram calculadas razões de prevalências (RP), intervalos de confiança (IC) de 95\% e os valores de $p$ pelo teste qui-quadrado. O nível de significância adotado foi de 5\%. Para a definição dos grupos de expostos e não expostos considerou-se como base o aporte da literatura científica.

A análise multivariada teve o propósito de descrever o efeito simultâneo das variáveis de interesse nos níveis de insatisfação. Foi empregada a técnica de regressão logística por se tratar de uma variável com desfecho dicotômico: satisfação (sim/não). Na seleção das variáveis para a análise multivariada foi empregado o teste do qui-quadrado de Pearson $\left(\mathrm{X}^{2}\right)$, considerando-se todas as variáveis de modo não condicional. O nível de significância para entrada no modelo multivariado foi de $25 \%$, com utilização do teste de razão de verossimilhança. Em seguida, procedeu-se ao método backward, adotando o critério de significância de 5\% para permanência no modelo final. A existência de colinearidade entre as variáveis preditoras do modelo foi testada por meio do variance inflation factor (VIF) ${ }^{16}$.

Finalmente, com base no modelo final obtido, sabendo que o modelo de regressão logística produz medidas de odds ratio (OR), empregou-se a regressão de Poisson, com variância robusta para estimar as $\mathrm{RP}$, intervalos de confiança de $95 \%$ e valores de $p^{17}$. O diagnóstico do modelo foi feito pelo teste de bondade 
de ajuste (Hosmer e Lemeshow), Curva ROC e pelo padrão de distribuição dos dados.

Foram empregados programas Statistical Program for the Social Sciences 15.0 (SPSS), OpenEpi 3.03a e o Software for Statistics and Data Science 12.0 (Stata).

Foram respeitados os requisitos da Resolução 466/2012, do Conselho Nacional de Saúde, com aprovação do Comitê de Ética em Pesquisa, sob o número de protocolo: 081/2009 (CAE 0086.0.059.000-09).

\section{Resultados}

Dos/as 3.084 participantes do estudo multicêntrico, perdeu-se informação de sete indivíduos, referente à variável sexo, incluindo-se neste estudo, 3.077 trabalhadores(as). Destes, $84,5 \%$ atuavam na atenção básica e 78,2\% eram mulheres, dentre as quais predominaram as jovens (58\%), pretas e pardas (84\%), com companheiro/a (56,8\%) e com filhos/as $(70,1 \%)$. Quanto à categoria profissional, prevaleceram as agentes comunitárias de saúde $(33,5 \%)$ e outras relacionadas à assistência indireta $(32,4 \%)$ que englobou os/as seguintes profissionais: pessoal administrativo, técnicas de nível médio, vigilantes, motoristas e trabalhadoras de serviços gerais. Entre os homens essas características também foram mais prevalentes, com exceção da categoria profissional na qual 11,7\% deles eram médicos (Tabela 1). A insatisfação global com o trabalho entre os homens foi de $27,4 \%$, e, entre as mulheres, foi um pouco menor, 25,6\% (Figura 1).

Na análise bivariada, a insatisfação com o trabalho associou-se, a níveis estatisticamente significantes, aos fatores sociodemográficos e às características do trabalho tanto para mulheres como para os homens (Tabelas 2 e 3 ).

Tabela 1 Características sociodemográficas de trabalhadores/as de saúde em serviços de média complexidade e de atenção básica. Bahia, Brasil, 2012

\begin{tabular}{|c|c|c|c|c|}
\hline \multirow{2}{*}{ Características Sociodemográficas } & \multicolumn{2}{|c|}{ Mulheres } & \multicolumn{2}{|c|}{ Homens } \\
\hline & $n$ & $\%$ & $n$ & $\%$ \\
\hline \multicolumn{5}{|l|}{ Idade* } \\
\hline Até 40 anos & 1.384 & 58 & 396 & 59,1 \\
\hline Mais de 40 anos & 1.004 & 42 & 274 & 40,9 \\
\hline \multicolumn{5}{|l|}{ Cor da Pele Autorreferida* } \\
\hline Pretos/as e pardos/as & 1.938 & 84 & 503 & 78,6 \\
\hline Brancos/as & 370 & 16 & 137 & 21,4 \\
\hline \multicolumn{5}{|l|}{ Situação Conjugal* } \\
\hline Com companheiro/a & 1.364 & 56,8 & 393 & 58,7 \\
\hline Sem companheiro/a & 1.037 & 43,2 & 276 & 41,3 \\
\hline \multicolumn{5}{|l|}{ Escolaridade* $^{*}$} \\
\hline Nível superior & 645 & 27,1 & 209 & 31,6 \\
\hline Nível técnico & 452 & 19,1 & 59 & 8,9 \\
\hline Nível médio e fundamental & 1.280 & 53,8 & 393 & 59,5 \\
\hline \multicolumn{5}{|l|}{ Tem Filhos* } \\
\hline Sim & 1.677 & 70,1 & 428 & 63,9 \\
\hline Não & 715 & 29,9 & 242 & 36,1 \\
\hline \multicolumn{5}{|l|}{ Número de Filhos** } \\
\hline Até 2 filhos & 1.211 & 58,1 & 281 & 47,4 \\
\hline Mais de 2 filhos & 874 & 41,9 & 312 & 52,6 \\
\hline \multicolumn{5}{|l|}{ Renda* } \\
\hline Até 2 salários mínimos & 1.464 & 73,8 & 339 & 59,3 \\
\hline Mais de 2 salários mínimos & 520 & 26,2 & 233 & 40,7 \\
\hline \multicolumn{5}{|l|}{ Categoria Profissional* } \\
\hline Enfermeiro/a & 188 & 7,9 & 25 & 3,7 \\
\hline Médico/a & 68 & 2,9 & 78 & 11,7 \\
\hline Outros/as de nível superior & 169 & 7,1 & 59 & 8,9 \\
\hline Técnico/a de enfermagem & 387 & 16,2 & 25 & 3,8 \\
\hline Agente comunitário de saúde & 804 & 33,5 & 126 & 19 \\
\hline Outros/as (assistência indireta) & 784 & 32,4 & 355 & 52,9 \\
\hline Total & 2.405 & 78,2 & 672 & 21,8 \\
\hline
\end{tabular}

*Variáveis com dados faltantes. 


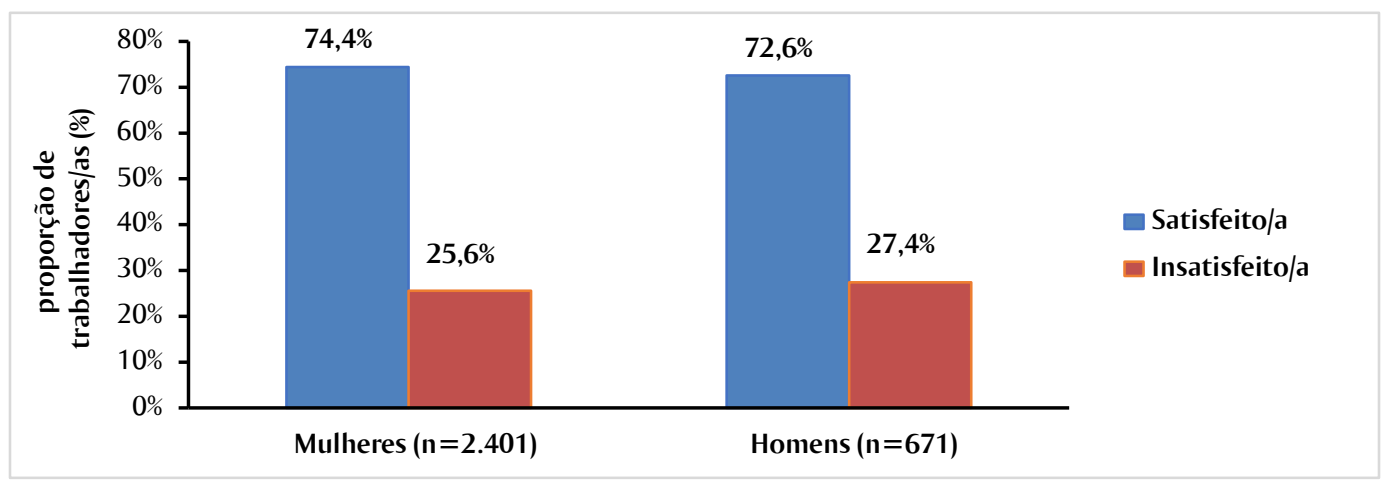

Figura 1 Insatisfação com o trabalho entre trabalhadores/as de saúde em serviços de média complexidade e de atenção básica. Bahia, Brasil, 2012

Tabela 2 Níveis de insatisfação com o trabalho segundo características sociodemográficas entre trabalhadores/as de saúde em serviços de média complexidade e de atenção básica. Bahia, Brasil, 2012

\begin{tabular}{|c|c|c|c|c|c|c|c|c|}
\hline \multirow{2}{*}{ Características Sociodemográficas } & \multicolumn{4}{|c|}{ Mulheres } & \multicolumn{4}{|c|}{ Homens } \\
\hline & Insatisfeita & $\%$ & $R P$ & IC (95\%) & Insatisfeito & $\%$ & $R P$ & IC $(95 \%)$ \\
\hline \multicolumn{9}{|l|}{ Idade* } \\
\hline Até 40 anos & 395 & 28,6 & 1,31 & $1,14-1,52$ & 137 & 34,6 & 2,01 & $1,49-2,69$ \\
\hline Mais de 40 anos & 218 & 21,8 & & & 47 & 17,2 & & \\
\hline \multicolumn{9}{|l|}{ Cor da Pele" } \\
\hline Pretos/as e pardos/as & 502 & 25,9 & 1,15 & $0,93-1,41$ & 139 & 27,6 & 1,02 & $0,75-1,39$ \\
\hline Brancos(as) & 83 & 22,6 & & & 37 & 27 & & \\
\hline \multicolumn{9}{|l|}{ Situação Conjugal } \\
\hline Com companheiro(a) & 340 & 25 & & & 95 & 24,2 & & \\
\hline Sem companheiro(a) & 275 & 26,6 & 1,06 & $0,93-1,22$ & 89 & 32,4 & 1,34 & $1,05-1,71$ \\
\hline \multicolumn{9}{|l|}{ Escolaridade* $^{*}$} \\
\hline Nível superior & 209 & 32,6 & & & 46 & 22 & & \\
\hline Nível técnico & 103 & 22,8 & 0,69 & $0,57-0,86$ & 22 & 37,3 & 1,69 & $1,12-2,57$ \\
\hline Nível médio e fundamental & 294 & 23 & 0,70 & $0,61-0,82$ & 113 & 28,8 & 1,31 & $0,97-1,76$ \\
\hline \multicolumn{9}{|l|}{ Tem Filhos* } \\
\hline Sim & 400 & 23,9 & & & 94 & 22 & & \\
\hline Não & 214 & 30 & 1,26 & $1,09-1,44$ & 90 & 37,3 & 1,70 & $1,36-2,17$ \\
\hline \multicolumn{9}{|l|}{ Número de Filhos* } \\
\hline Até 2 filhos & 304 & 25,1 & & & 71 & 25,3 & & \\
\hline Mais de 2 filhos & 233 & 26,7 & 1,06 & $0,92-1,23$ & 88 & 28,3 & 1,12 & $0,86-1,46$ \\
\hline \multicolumn{9}{|l|}{ Renda* } \\
\hline Até 2 salários mínimos & 350 & 23,9 & 0,88 & $0,74-1,04$ & 98 & 28,9 & 1,20 & $0,91-1,59$ \\
\hline Mais de 2 salários mínimos & 141 & 27,2 & & & 56 & 24 & * & \\
\hline \multicolumn{9}{|l|}{ Categoria Profissional* } \\
\hline Médico(a) & 17 & 25,4 & & & 16 & 20,5 & & \\
\hline Enfermeiro(a) & 63 & 33,9 & 1,33 & $0,84-2,10$ & 3 & 12 & 0,58 & $0,18-1,84$ \\
\hline Outros/as de nível superior & 46 & 27,2 & 1,07 & $0,66-1,73$ & 16 & 27,1 & 1,32 & $0,72-2,42$ \\
\hline Técnico(a) de enfermagem & 87 & 22,5 & 0,88 & $0,56-1,39$ & 8 & 32 & 1,56 & $0,76-3,20$ \\
\hline ACS & 196 & 24,4 & 0,96 & $0,63-1,47$ & 39 & 31 & 1,51 & $0,91-2,51$ \\
\hline Outros(as) & 204 & 26 & 1,02 & $0,67-1,57$ & 102 & 28,8 & 1,40 & $0,88-2,24$ \\
\hline Total & 615 & 25,6 & & & 184 & 27,4 & & \\
\hline
\end{tabular}

P: Prevalência; RP: razões de prevalências; IC: intervalos de confiança.

"Variáveis com dados faltantes. 
Tabela 3 Níveis de insatisfação com o trabalho segundo características laborais entre trabalhadores/as de saúde em serviços de média complexidade e de atenção básica. Bahia, Brasil, 2012

\begin{tabular}{|c|c|c|c|c|c|c|c|c|}
\hline \multirow{2}{*}{$\begin{array}{c}\text { Características } \\
\text { Sociodemográficas }\end{array}$} & \multicolumn{4}{|c|}{ Mulheres } & \multicolumn{4}{|c|}{ Homens } \\
\hline & Insatisfeita & $\%$ & $R P$ & IC (95\%) & Insatisfeito & $\%$ & $R P$ & IC (95\%) \\
\hline \multicolumn{9}{|l|}{$\begin{array}{l}\text { Tempo de trabalho } \\
\text { na profissão* }\end{array}$} \\
\hline Até 5 anos & 257 & 25,4 & 0,98 & $0,85-1,12$ & 85 & 28 & 1,03 & $0,80-1,32$ \\
\hline Mais de 5 anos & 356 & 26 & & & 99 & 27,2 & & \\
\hline \multicolumn{9}{|l|}{ Vínculo de trabalho*" } \\
\hline Efetivo & 438 & 27,4 & & & 124 & 32,9 & & \\
\hline Temporário & 168 & 21,4 & 0,78 & $0,66-091$ & 56 & 19,6 & 0,59 & $0,45-0,79$ \\
\hline \multicolumn{9}{|l|}{ Turno de trabalho** } \\
\hline Diurno & 473 & 25 & & & 148 & 27,5 & & \\
\hline Noturno/regime de plantão & 130 & 27,1 & 1,08 & $0,92-1,28$ & 34 & 26 & 0,94 & $0,69-1,29$ \\
\hline \multicolumn{9}{|l|}{$\begin{array}{l}\text { Tempo de trabalho } \\
\text { na unidade* }\end{array}$} \\
\hline Até 2 anos & 91 & 22,1 & 0,83 & $0,68-1,01$ & 50 & 23 & 0,79 & $0,59-1,05$ \\
\hline Mais de 2 anos & 524 & 26,6 & & & 124 & 29,2 & & \\
\hline \multicolumn{9}{|l|}{$\begin{array}{l}\text { Fez treinamento para } \\
\text { exercer o cargo" }\end{array}$} \\
\hline Sim & 340 & 22,6 & & & 85 & 24,5 & & \\
\hline Não & 297 & 27,9 & 1,16 & $1,01-1,33$ & 99 & 29,6 & 1,11 & $0,90-1,49$ \\
\hline \multicolumn{9}{|l|}{ Tem recebido treinamento* } \\
\hline Sim & 340 & 22,6 & & & 76 & 22,6 & & \\
\hline Não & 268 & 31 & 1,37 & $1,19-1,57$ & 106 & 32,6 & 1,45 & $1,12-1,86$ \\
\hline \multicolumn{9}{|l|}{$\begin{array}{l}\text { Atividades compatíveis } \\
\text { com o cargo"* }\end{array}$} \\
\hline Sim & 548 & 24,3 & & & 157 & 25,7 & & \\
\hline Não & 67 & 49,6 & 2,04 & $1,69-2,46$ & 27 & 46,6 & 1,81 & $1,33-2,46$ \\
\hline \multicolumn{9}{|l|}{ Direitos trabalhistas* } \\
\hline Sim & 574 & 25,7 & & & 170 & 27,3 & & \\
\hline Não & 32 & 24,6 & 0,96 & $0,70-1,30$ & 12 & 33,3 & 1,22 & $0,75-1,97$ \\
\hline \multicolumn{9}{|l|}{ Possuir outro trabalho* } \\
\hline Sim & 472 & 25,8 & & & 56 & 26,7 & 0,93 & $0,71-1,21$ \\
\hline Não & 136 & 24,8 & 0,96 & $0,81-1,13$ & 123 & 28,8 & & \\
\hline \multicolumn{9}{|l|}{$\begin{array}{l}\text { Jornada semanal de } \\
\text { trabalho total* }\end{array}$} \\
\hline Até 40 horas & 488 & 26,1 & & & 123 & 27,4 & & \\
\hline Mais de 40 horas & 112 & 27,7 & 1,06 & $0,79-1,13$ & 57 & 27,5 & 1,01 & $0,77-1,31$ \\
\hline Total & 615 & 25,6 & & & 184 & 27,4 & & \\
\hline
\end{tabular}

P: Prevalência; RP: razões de prevalências; IC: intervalos de confiança.

*Variáveis com dados faltantes.

Entre as mulheres, após a regressão logística, permaneceram no modelo final os seguintes fatores: possuir até 40 anos de idade, nível técnico de escolaridade, nível médio/ fundamental de escolaridade, vínculo de trabalho temporário e exercer atividades não compatíveis com o cargo (Tabela 4). As variáveis "possuir até 40 anos de idade" (RP: 1,36; IC 95\%: 1,17-1,58) e "exercer atividades não compatíveis com o cargo" (RP: 2,17; IC 95\%: 1,81-2,60) elevaram os níveis de insatisfação, enquanto a insatisfação entre aquelas com nível técnico (RP: 0,70; IC 95\%: 0,58-0,86), nível médio/ fundamental (RP: 0,66; IC 95\%: 0,57-0,77) e vínculo de trabalho temporário (RP: 0,66; IC 95\%: 0,56-0,77) foi menor, em níveis estatisticamente significantes. 
Tabela 4 Insatisfação com o trabalho entre trabalhadores/as de saúde em serviços de média complexidade e de atenção básica. Bahia, Brasil, 2012

\begin{tabular}{lcccc}
\hline & \multicolumn{3}{c}{ Insatisfação com o Trabalho } \\
\cline { 2 - 5 } Características Sociodemográficas & \multicolumn{2}{c}{ Mulheres $(n=2332)$} & \multicolumn{2}{c}{ Homens $(n=658)$} \\
& $R P$ & $I C(95 \%)$ & $R P$ & $I C(95 \%)$ \\
\hline Idade $<40$ anos & 1,36 & $1,17-1,58$ & 2,09 & $1,56-2,81$ \\
Nível médio/ fundamental* & 0,70 & $0,58-0,86$ & - & - \\
Nível técnico* & 0,66 & $0,57-0,77$ & 1,74 & $1,30-2,34$ \\
Atividades não compatíveis com o cargo & 2,17 & $1,81-2,60$ & 0,57 & $0,44-0,74$ \\
Vínculo de trabalho temporário & 0,66 & $0,56-0,77$ & & - \\
\hline
\end{tabular}

RP: razões de prevalências; IC: intervalos de confiança.

*As variáveis nível médio/fundamental e nível técnico de escolaridade não permaneceram no modelo final de regressão logística, no estrato masculino.

$\mathrm{Na}$ análise de regressão logística, entre os homens, três variáveis permaneceram no modelo: possuir até 40 anos de idade, exercer atividades não compatíveis com o cargo e vínculo de trabalho temporário (Tabela 4). Semelhante ao observado entre as mulheres, as variáveis "possuir até 40 anos de idade" (RP: 2,09; IC 95\%: 1,56-2,81) e "exercer atividades não compatíveis com o cargo" (RP: 1,74; IC 95\%: 1,30-2,34) elevaram os níveis de insatisfação, enquanto "ter vínculo de trabalho temporário" (RP: 0,57; IC 95\%: 0,44-0,74) registrou menor prevalência de insatisfação.

Não foi verificada colinearidade entre as variáveis preditoras do modelo, sendo o valor de VIF menor do que $10^{15}$, nos dois estratos de gênero. Contudo, fato curioso ocorreu em relação à distribuição da variável "escolaridade", comportando-se de modo diverso segundo o gênero. Observou-se, entre as mulheres, maior grau de insatisfação naquelas com maior nível de escolaridade, e as variáveis "nível técnico" e "nível médio/ fundamental" de escolaridade se comportaram como fatores associados à menor prevalência de insatisfação. Nos estratos masculinos de escolaridade, por sua vez, verificou-se maior insatisfação entre aqueles que possuíam menor nível de escolaridade.

Os modelos finais obtidos foram analisados quanto à adequação dos dados. Para o diagnóstico dos modelos, foram analisadas: a bondade de ajuste do modelo, através do teste de Hosmer e Lemeshow, níveis de sensibilidade e especificidade, por meio da Curva ROC, e o padrão de distribuição dos dados, para verificação da existência de dados influentes.

Entre as mulheres, no modelo final obtido, a hipótese alternativa de que o modelo não se adequava aos dados foi rejeitada (teste de Hosmer e Lemeshow revelou $p=0,150$ ). A Curva ROC revelou uma área igual a 0,62. Na análise do padrão das covariáveis para verificação da influência das observações extremas, a comparação dos modelos com e sem as observações demonstrou pouca influência, não havendo mudança significante nos coeficientes obtidos.
Entre os homens, o modelo final também se revelou ajustado aos dados. O teste de Hosmer e Lemeshow indicou $p=0,96$, sendo rejeitada a hipótese alternativa. A Curva ROC revelou uma área igual a 0,67. A ocorrência de dados influentes que alterassem o ajuste do modelo não foi evidenciada. Desta forma, os modelos obtidos demonstraram estar bem ajustados aos dados, sendo mantidos.

\section{Discussão}

A elevada feminização da equipe de saúde, mais expressiva nas profissões ligadas à assistência direta aos usuários (enfermagem, técnicas de enfermagem, agente comunitária de saúde - ACS), reitera a discussão já presente na literatura ${ }^{1}$. Verifica-se a reprodução das tarefas que ocorrem no âmbito doméstico, com profissões voltadas para o cuidado, decorrente do estereótipo feminino, socialmente construído e cooptado pelo mercado de trabalho ${ }^{18}$.

Os níveis de insatisfação variaram entre homens e mulheres, conforme as características sociodemográficas e do trabalho. Em ambos os estratos, associaram-se positivamente à insatisfação os seguintes fatores: idade menor de 40 anos e executar atividades não compatíveis com o cargo.

Verificou-se que a insatisfação com o trabalho diminuiu conforme aumentou a idade, sendo os adultos jovens mais expostos a alta insatisfação. $\mathrm{O}$ achado de insatisfação entre os/as mais jovens pode estar relacionado à inexperiência profissional e incipiente habilidade instrumental, associados à descrença da equipe e dos/as pacientes em relação à competência desses/as profissionais, muitas vezes, recém-formados. Isso gera estresse e dificuldade nas relações interpessoais com a equipe (muitas vezes resistente) e com os/as usuários/ as dos serviços de saúde ${ }^{9}$.

Em contrapartida, o maior grau de satisfação entre os mais velhos pode ser explicado pelo fato de que o maior tempo de trabalho na profissão favorece 
a aquisição de experiência para lidar com as situações de estresse no dia a dia do trabalho, assim como melhor adaptação ao emprego. Desse modo, os mais velhos podem considerar aspectos geralmente desagradáveis aos mais jovens (causadores de insatisfação) como fatores positivos para o desenvolvimento do trabalho, a exemplo da relação interpessoal com a equipe e com a chefia. Trata-se, portanto, de um processo adaptativo, no qual aqueles/as que conseguiram criar estratégias mais bem-sucedidas para atender às demandas tendem a permanecer no emprego ${ }^{4}$. A experiência de lidar ou contornar situações difíceis pode ser favorecidas pela idade, ajudando a reduzir insatisfação que tais situações produziriam.

Executar atividades não compatíveis com o cargo contribuiu para elevar os níveis de insatisfação entre homens e mulheres, fato também observado em outros estudos ${ }^{19,20}$. O acúmulo de atividades pode ser decorrente da insuficiência de pessoal e de recursos materiais associados às péssimas condições de trabalho ${ }^{19}$. Na tentativa de prestar um cuidado qualificado, o/a trabalhador/a se sujeita a desenvolver inúmeras funções, por vezes incompatíveis com o cargo ocupado. Ao deparar-se com uma situação de sobrecarga de atividades associadas às condições precárias de trabalho e diante da impossibilidade de efetivar um cuidado qualificado, elevam-se os níveis de insatisfação dos/as trabalhadores $/ \mathrm{as}^{20}$.

O vínculo de trabalho temporário associou-se negativamente à insatisfação com o trabalho entre homens e mulheres. O predomínio da insatisfação entre os/as trabalhadores/as com vínculo efetivo de trabalho apontou na direção oposta ao esperado, visto que este tipo de vínculo deveria, em princípio, estar associado à maior satisfação no trabalho, uma vez que envolve maior segurança laboral e maior estabilidade financeira, resultando, ainda, em benefícios acrescentados ao salário e garantia de direitos trabalhistas, situação diversa daquela identificada entre os/as trabalhadores/as com vínculo temporário. Diante disso, acredita-se que o resultado inesperado pode estar relacionado ao medo de sofrer represálias por parte da chefia, de modo que trabalhadores/as com vínculo temporário poderiam fornecer respostas supostamente esperadas deles/as, apesar da garantia de confidencialidade das informações. Contudo, este dado precisa ser analisado mais detalhadamente para sua adequada compreensão.

Apesar do vínculo de trabalho estável, os/as trabalhadores/as de saúde estão inseridos em um cenário ocupacional desmotivador. Ressalta-se que às condições de trabalho precárias - relacionadas à estrutura física das unidades, déficit de recursos materiais, baixos salários, jornada de trabalho excessiva, sobrecarga de trabalho e dimensionamento insuficiente de pessoal - soma-se a ausência de perspectiva de ascender na profissão, em virtude de não haver plano de cargos e salários que considere o tempo de profissão e o nível de formação desses/as profissionais ${ }^{19,21}$.

Deve-se ainda considerar que o vínculo efetivo de trabalho predominou entre trabalhadores/as que exerciam profissões de níveis técnico e médio/ fundamental (ACS, técnicos/as de enfermagem e outras relacionados à assistência indireta), podendo ser estas profissões as mais desvalorizadas, mal remuneradas e expostas a piores condições de trabalho. Diante destas questões, possuir um vínculo de trabalho estável não foi suficiente para garantir a satisfação ocupacional. Nessa perspectiva, observa-se a necessidade de adotar e implementar medidas que valorizem esses/as profissionais em suas práticas diárias.

A escolaridade associou-se à insatisfação com o trabalho, entre as mulheres, a níveis estatisticamente significantes, permanecendo no modelo final obtido na regressão logística. Foi evidenciada redução da insatisfação entre as trabalhadoras com menor nível de escolaridade (nível técnico e nível médio/ fundamental).

O menor grau de insatisfação observado entre trabalhadoras com nível técnico e fundamental/ médio de escolaridade pode estar associado à menor responsabilidade na tomada de decisões para manutenção e funcionamento dos serviços, tendo em vista que, muitas vezes, o gerenciamento da unidade é delegado aos profissionais de nível superior, a exemplo das enfermeiras, categoria profissional que apresentou maior grau de insatisfação no estrato feminino ${ }^{10}$. Presume-se, ainda, que as trabalhadoras com escolaridade mais elevada possuam maiores expectativas em relação ao trabalho. Quando os ganhos advindos da atividade laboral não condizem com as expectativas das trabalhadoras, emerge o sentimento de insatisfação ${ }^{22}$.

A variável "escolaridade”, apesar de não permanecer no modelo final de regressão logística no estrato masculino, comportou-se de forma oposta quando comparados homens e mulheres. A análise bivariada evidenciou que, entre os homens, quanto maior o grau de escolaridade menor era o nível de insatisfação. O oposto ocorreu entre as mulheres, sendo a insatisfação com o trabalho diretamente proporcional ao grau de escolaridade, ou seja, quanto maior o nível de escolaridade, maior foi a insatisfação com o trabalho.

O aumento do nível de escolaridade permitiu às mulheres participar mais fortemente nas atividades econômicas, políticas e educacionais, o que é um ganho importante para disseminação de valores de igualdade nas questões de gênero. Contudo, apesar 
das mudanças na posição social das mulheres e sua maior inserção no mercado de trabalho, o ideal igualitário ainda não foi alcançado, persistindo a divisão sexual do trabalho, que expõe as mulheres a atividades de trabalho mais precárias, com menor remuneração, e menos reconhecidas socialmente ${ }^{23}$, apesar do nível de escolaridade mais elevado. A percepção da desigualdade de gênero, persistente no trabalho, pode ser fonte de insatisfação entre as mulheres com maior escolaridade, justificando as diferenças encontradas, nos níveis de insatisfação com o trabalho, entre homens e mulheres com mesmo grau de escolaridade.

A base sobre a qual se estabeleceu e é perpetuada a divisão sexual do trabalho na saúde remete ao processo de valorização da qualificação de homens e mulheres para o exercício das profissões. Enquanto as qualidades necessárias aos homens para as profissões ditas masculinas são institucionalizadas no âmbito público, as qualidades necessárias às mulheres para exercer as profissões ditas femininas são aprendidas no âmbito privado (o cuidado, a passividade, a generosidade, por exemplo), sendo desprovidas de valor e reconhecimento social ${ }^{24}$, apesar de serem incorporadas pelo mercado de trabalho.

A construção da identidade profissional baseada em qualidades de sexo é o combustível para a perpetuação de relações desiguais de gênero, visto que se encontra ancorada em um sistema hierarquizado que valoriza a qualificação masculina na esfera pública e menospreza as qualidades "femininas", adquiridas no âmbito privado, ao tempo que se estabelecem critérios para valorização profissional, remuneração e reconhecimento social do trabalho realizado por homens e mulheres ${ }^{25}$. Esse contexto explica os mecanismos de exploração e de subordinação do trabalho feminino, bem como os elevados níveis de insatisfação entre as mulheres, apesar do maior grau de escolaridade.

Ressaltamos que, apesar do estudo alcançar uma amostra relativamente grande, quando estratificada por sexo, resultou em uma porcentagem pequena de trabalhadores do sexo masculino, o que pode ter comprometido a investigação de diferenças relevantes nos níveis de insatisfação com o trabalho, entre os grupos. Deve-se ainda considerar os vieses inerentes aos estudos transversais.

No entanto, o estudo traz à tona achados importantes, que demonstram a persistência de assimetrias relacionadas ao gênero e à divisão sexual do trabalho e seus impactos nos níveis de satisfação laboral.

\section{Conclusão}

Homens e mulheres vivenciam elevados níveis de insatisfação com o trabalho e características pessoais e laborais permaneceram associadas à insatisfação, nos estratos masculino e feminino (idade, vínculo de trabalho, exercer atividades não compatíveis com o cargo). O nível de escolaridade, no entanto, impactou de forma diferenciada a insatisfação com o trabalho nos estratos, revelando a persistência de desigualdades de gênero e a divisão sexual do trabalho, sendo as mulheres mais expostas às atividades mais precárias, de menor remuneração e com menor reconhecimento social. Um melhor entendimento desses aspectos pelos gestores pode subsidiar o planejamento e execução de políticas e estratégias que busquem minimizar os efeitos desses fatores sobre os/as trabalhadores/as e contribuir para a construção de ambientes de trabalho mais saudáveis.

É preciso avançar no que se refere às constatações das desigualdades nos estudos de gênero, desnaturalizando os achados e destacando as relações de poder que ainda resistem nos ambientes de trabalho. Diante do exposto, torna-se relevante considerar que o gênero interfere de forma significativa na escolha da profissão, na inserção no mercado de trabalho e nas possibilidades de ascensão profissional. Com isso, será possível promover medidas que reduzam as assimetrias de gênero relativas à insatisfação com o trabalho e que, de modo geral, elevem os níveis de satisfação laboral dos homens e das mulheres.

\section{Contribuições de autoria}

Sousa CC contribuiu nas diversas fases da investigação e da redação do artigo, as quais incluíram coleta e análise de dados, redação e revisão do texto; Araújo TM contribuiu na coleta, análise de dados e revisão do texto; Pinho PS e Freitas AMC contribuíram na interpretação de resultados e na elaboração e redação do texto. Todos os autores participaram na aprovação do texto para publicação e assumem responsabilidade integral por seu conteúdo. 


\section{Referências}

1. Bacha AM, Grassioto OR, Cacique DB, Carvasan, GAF, Machado HC. Satisfação no trabalho no contexto hospitalar: uma análise segundo o gênero. Esc Anna Nery. 2015;19(4):549-56.

2. Locke EA. What is job satisfaction? Organ Behav Hum Perform. 1969;4(4):309-36.

3. Ovadje F. Exploring turnover among middle managers in a non-western context. IJBR. 2009;10(2):64-80.

4. Carrilo-Garcia C, Solano-Ruíz MDC, MartinezRoche ME. Gomez-Garcia CI. Influência do gênero e da idade: satisfação no trabalho de profissionais da saúde. Rev Lat Am Enfermagem. 2013;21(6):1314-20.

5. Saletti-Cuesta L, Delgado A, Ortiz-Gómez T, López-Fernández LA. Diferencias de género en la percepción del logro profesional en especialistas de medicina familiar y comunitaria.

Rev Esp Salud Publica. 2013;87(3);221-38.

6. Sloanne P, Williams H. Job satisfaction, comparison, earnings and gender. Labour. 2000;14(1):473-502.

7. Clark AE. Job satisfaction and gender: why are women so happy at work? Labour Econ. 1997;4(4):341-418.

8. Atif K, Khan HU, Maqboll S. Job satisfaction among doctors, a multi-faceted subject studied at a tertiary care hospital in lahore. Pak J Med Sci. 2015;31(3):610-4

9. Ribeiro RBN, Assunção AA, Araújo, TM. Factors associated with job satisfaction among publicsector physicians in Belo Horizonte, Brazil. Int J Health Serv. 2014;4(1):787-804.

10. Ishara S, Bandeira M, Zuardi AW. Public psychiatric services: job satisfaction evaluation. Braz J Psychiatry. 2007;30(1):38-41.

11. Kifle T, Desta IH. Gender differences in domains of job satisfaction: evidence from doctoral graduates from Australian Universities. Econ Anal Policy. 2012;42(3):319-38.

12. Fadel CB, Carvalho ML, Arcieri RM, Saliba NA, Garbin CAS. Interesse e satisfação profissional de quem atua em equipes do programa saúde da família no noroeste paulista. REME. 2008;12(1):64-70.

13. Lapischies SRC, Jardim VMR, Kantorski LP. Fatores associados à satisfação no trabalho em Centros de
Atenção Psicossocial. Rev Lat Am Enfermagem. 2014;22(6):950-8.

14. Wanous JP, Reichers AE, Hudy MJ. Overall job satisfaction: how good are single-item measures? J Appl Psychol. 1997;82(2):247-52.

15. Ommen O, Driller E, Köler T, Kowalski C, Ernstmann N, Neumann M, et al. The relationship between social capital in hospitals and physician job satisfaction. BMC Health Serv Res. 2009;9:81.

16. Hair JF Jr, Black WC, Babi HJ, Anderson RE, Tatham RL. Análise multivariada de dados. 5th ed. Porto Alegre: Bookman; 2005.

17. Coutinho LMS, Scazufca M, Menezes PR. Métodos para estimar razão de prevalência em estudos de corte transversal. Rev Saude Publica. 2008;42(6):992-8.

18. Barbosa RHS. Gender and healthcare work: a critical view of community healthagents' work. Interface Comun Saude Educ. 2012;16(42):751-65.

19. Lima L, Pires DEP, Forte ECN, Medeiros F. Satisfação e insatisfação no trabalho de profissionais de saúde da atenção básica. Esc Anna Nery. 2014;18(1):17-24.

20. Forte ECN, Pires DEP. Enfermeiras na atenção básica: entre a satisfação e a insatisfação no trabalho. Trab Educ Saude. 2017;15(3):709-24.

21. Soratto J, Pires DEP, Trindade LL, Oliveira JS, Forte ECN, Melo TP. Insatisfação no trabalho de profissionais da saúde na estratégia saúde da família. Texto Contexto Enferm. 2017;26(3):e2500016.

22. Guedes SAG, Oliveira CCC, Albuquerque RLC Jr. Análise da satisfação dos profissionais de saúde da família com as condições de trabalho. Cien Cuid Saude. 2013;12(1):121-30.

23. Sorj B. Percepções sobre tarefas separadas de gênero. In: Araújo C, Scalon C., organizadores. Gênero, família e trabalho no brasil. Rio de Janeiro: Editora FGV; 2005. p. 79-88.

24. Kergoat D. Da divisão do trabalho entre os sexos. Tempo Social. 1989;1(2):73-103.

25. Araújo TM, Rotenberg L. Relações de gênero no trabalho em saúde: a divisão sexual do trabalho e a saúde dos trabalhadores. In: Assunção AA; Brito J., organizadores. Trabalhar na saúde: experiências cotidianas e desafios para a gestão do trabalho e do emprego. Rio de Janeiro: Editora Fiocruz, 2011. p. 131-150. 